UCRL-YC-117613

PREPRINT

\title{
Magnetic X-Ray Circular Dichroism in Spin-Polarized Photcelectron Diffraction
}

\author{
G.D. Waddill \\ J.G. Tobin \\ $x$ Guo \\ S.Y. Tong
}

This paper was prepared for submittal to the Journal of Physica $B$ and XAFS8

Berlin, Germany

August 28-September 2, 1994

July 12, 1994

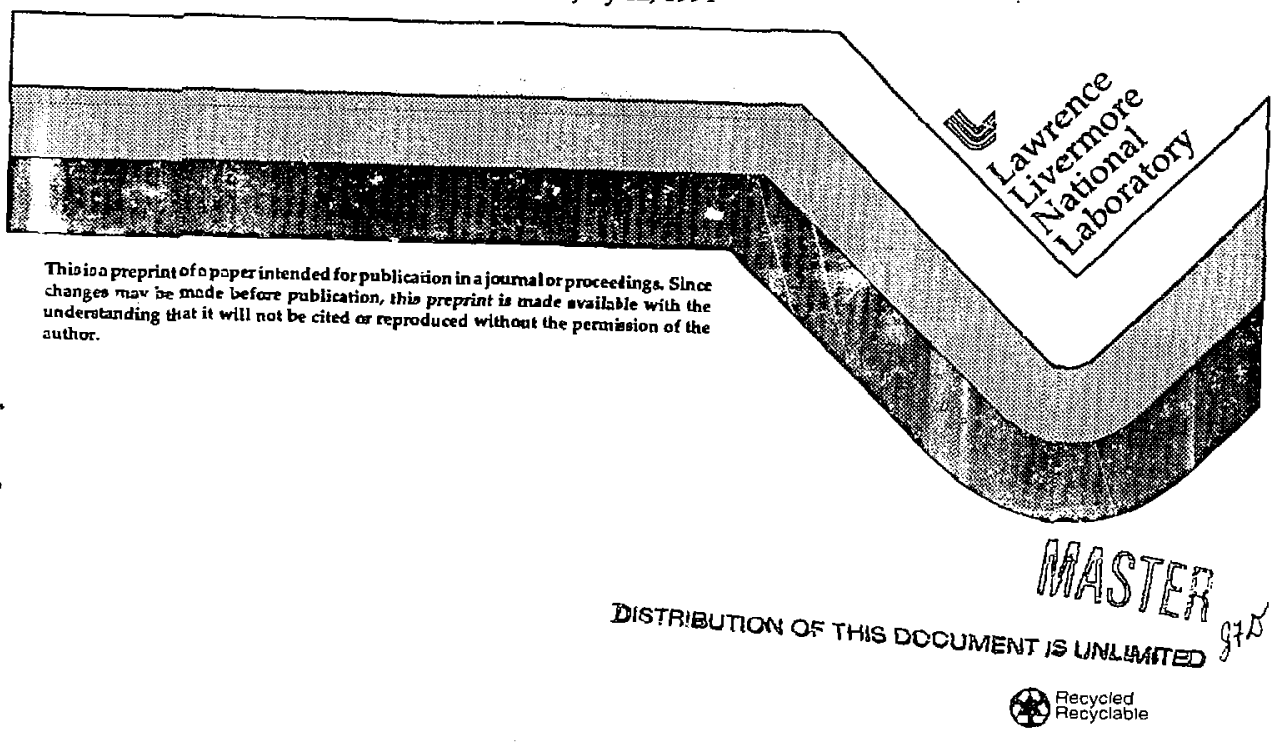




\section{DISCLAIMER}

This document was prepared as an account of work sponsored by an agency of the Libited Stotes Government. Neither the United Steles Government nor the Uni versity of California nor any of their employees, makes any warranty, express or implied, or assumes any legalligbility or responsibility for the accuracy, completeness, or usefulness of any information, apparatus, produch, or process disedosed, or represents that its use would not infringe privately owned rights. Refereme he rein to any specific commercial products, process, or service by trade name, trademank, manufacturer, or othervise, does not necessarily constitute orimply its endorsement, recommendation, or fovering by the United States Govemment or the University of California. The views and opinions of authors expressed herein do not necessarily stale or reflect thos: of the inited States Govermment or the Lniversity of Caljforvin, and shall not be used for advertising or product endorsement purposes. 


\title{
Magmetic X-Ray Circular Dichroism in Spin-Polarized Photoelectron Diffraction
}

\author{
G.D. Waddilla, J.G. Tobina, X. Guob, and S.Y. Tongb \\ aLawrence Livermore National Laboratory, Chemistry and Materials Science Department, P.O. \\ Box 808, L-357, Livermore, CA 94550 - USA \\ bUniversiy of Wisconsin-Milwaukee, Laboratory for Surface Studies and Department of Physics, \\ Milwaukee, MI 53201 - USA
}

\begin{abstract}
The first structiral determination with spin-polarized, energy-dependent photoelectron diffraction using circularly-polarized $x$-rays is reported for Fe films on $\mathrm{Cu}(001)$. Circularly-polarized $x$-rays produce spin-polarized photoelectrons from the Fe $2 \mathrm{p}$ doublet, and intensity asymmetries in the $2 p_{3 / 2}$ level are observed. Fully spin-specific multiple scattering calculations reproduce the experiment " $y$-determined energy and angular dependences. A new analytical procedure which focuses upon intensity variations due to spin-dependent diffraction is introduced. A sensitivity to local geometric and magnetic structure is demonstrated.
\end{abstract}

Keywords: MCXD, Photoelectron Diffraction, Magneric Structure

Corresponding Author: James G. Tobin

Lawrence Livermore Natiunal Laboratory

Post Office Box 808, L-357

Livermore, CA 94550 - USA

(510) 422-7247/Telephone

(5i0) 423-7040/Fax

E-Mail: TOBIN@CMSILLPJ.GOV 


\section{MANTEXI}

Determining the nanoscale structure-property relationships of a magnetic surface, ultrathin film or interface remains a formidable but potentially extraordinarily rewarding task. For example, as studies of giant magneto resistive ${ }^{[1]}$ and spin valve ${ }^{[2]}$ systems have progressed, it has become clear tha: spin-specific interfacial scattering is a crivical but poorly enderstood event. To pro $_{1}$ orly address such problems, a probe which combines elemental specificity with a sensitivity to local order, both geometric and macnetic, is essential. Spin-polarized photoelectron diffraction (SPPD) is potentially an ideal candidate for studying nanoscale magnetic systems, with the above attributes plus the promise of an ultimate extension to elemert-and spin-specific imaging ${ }^{[3]}$. However, while the potential for SPPD is very high, particularly with the advent of third generasion synchrozon rabiasion facilities such as the Advanced Light Sovree, until now a definitive demonstration has been lacking. This is primarily because of the relatively inefficient nature of spin-detectors and the relatively weak nature of magnetic scattering effects. Here we present a prototype study using magnetic $x$-ray circular dichroism $[4]$, to permit a direct and unambiguous control of electron poiarization without the necessity of the low-efficiency spindetectors. This is coupled with a fully spin-specific, muliple scattering computational analysis. While we remained hampered by the limitations of existing sources of circularly-polarized $x$-rays and hence are constrained in terms of signal to noise, this is the first definitive demonstration of SPPD.

In some respects, this SPPD investigation is an independent verification and extension of the pioneering studies of G. Schütz, et al. [5], who used spin-polarized EXAFS to probe bulk magnetic systems. Consistent with non-spin PD and EXAFS studies, the SPPD shows a larger effect: the SPPD escillations are on the onter of $2 \%$ white the Go metal SPEXAFS oscillations are $\leq 1 / 3 \%$. Additionally, SPPD has the advantage of both energy and angular variations, which is essential to the extension to photoelecton diffraction imaging[3]. Both this work and the ground-breaking studies of Schütz, et al. ${ }^{[5]}$ are predicated upon control of spin-polarization of ejected electrons via excitation with circularly-polarized $\mathbf{x}$-r2ys. In a simplistic picture, $2 \mathrm{p}$ photoemission total cross sections from ferromagnetic materials will exhibir 2 polarized distribution of $62.5 \%$ (37.5\%) minority spin electrons from the $2 \mathrm{p} 3 / 2$ and $25 \%(75 \%)$ minority spin electrons from the $2 \mathrm{p}_{1 / 2}$, when excitec with right (left) circularly-polarized radiation that is collinear with the magnetic axis of the sanple. These adjustably spin-polarized electrons can then scatter off of nearby neighbors, producing a sensirivity to both local geometric and magnetic ordering. (Although we have chosen to use a ferromagnetic system as a test case, these same selection rules will apply in genera', e.g., to paramagnetic and anti-ferromagnetic ordering, and the multiple scattering analysis should be sensitive to differences in the local order of each 
structure.) To avoid extraneous effects and to allow internal cross-checking of data, measurements were performed only in mirror planes, where only the relative alignment of the photon helicity and magnetization is crucial. Thus reversing the absolute value of these quantities, while maintaining the same relative spin orientation, serves as a convenient but absolurely essential consistency test to determine if the observed asymmetry is due to spindependent diffraction. It is the absence of such polarization control or electron spin-detection, plus the ill-defined nature of the intrinsic 3 s polarization, that has hampered previous antempts at SPPD using the $3 \mathrm{~s}$ level of $3 \mathrm{~d}$ transition metals $[6-8]$.

Recause the calculated spin-dependent intensities are for an entire $2 p_{3 / 2}$ or $2 p_{1 / 2}$ manifold, the best comparison with experiment uses an integrated spin-dependent intensity asymmetry (or integrated SIA) defined as follows:

Integrated SIA(hv) $=\frac{\left[\frac{I_{3 / 2}^{+}(h v)}{I_{1 / 2}^{+}(h v)}-\frac{I_{3 / 2}(h v)}{I_{1 / 2}^{-}(h v)}\right]}{\left[\frac{I_{3 / 2}^{+}(h v)}{I_{1 / 2}^{+}(h v)}+\frac{I_{3 / 2}^{-}(h v)}{I_{1 / 2}(h v)}\right]}$

where, for example, $I_{3 / 2}^{+}$is the parallel intensity integrated over the $2 p_{3 / 2}$ manifold. In Figure 1 , we show the calculated asymmetry (solid curve) along the [111] direction for a model with fccFe spacings ( $\mathrm{d}_{12}=1.8 \AA, \mathrm{d}_{23}=1.8 \AA$ ) ${ }^{[8]}$ as a function of photon energy compared to the asymmetry extracted from the experimental data. The integrated SLA gives a goos quantitative agreement with the calculations and even reproduces the sign change in the asymnetry $c a . h v=840 \mathrm{eV}$. The energy oscillations due to spin-dependent photoelectron diffraction are fundamental and are present even in the peak height intensities. Furthermore, variations of the structural parameters by $\pm 0.2 \AA$ destroys the agreement between the measurements and simulations. In fact, we have performed a detailed structural determination by varying the spatial parameters in the model and performing a quantitative $r$ factor analysis of calculated anr measured values. Our best fit occurs for $d_{12}=1.9 \AA$ and $d_{23}=1.7 \AA$ (dashed curve), with a Pendry $r$ factor $\left(r_{p}\right)$ of 0.17 , which is $9 \%$ better than the unrelaxed structure, a measurable difference. This is consistent with our previous determination [9] but exceeds those earlier results with a clear improvement in sensitivity to the small surface relaxations. Removal of sample remanent magnerization obliterated any efitch driving ISIA to zero. Thus, the agreement between theory and experiment indicates that the intensity asymmetry arises from spin-dependent diffraction and demonstrates a sensitivity to both local geometric and magnetic stucture. 
In summary, we have for the first time measured spin-dependent photoelectron diffraction using circularly-polarized $x$-rays. The resultant spin-polarized $2 \mathrm{p}$ photoemission peaks exhibit angle and energy dependent intensity variations due to spin-dependent final state diffraction. The results are well described by spin-polarized photoemission, multiple scantering calculations.

Work performed under the auspices of the U.S. Department of Energy by the Lawrence Livermore National Laboratory under contract number W-7405-ENG-48. SSRL is supported by the Chemical Sciences Division of DOE/BES. The work at the University of WisconsinMilwaukee was supported by DOE DE-FG02-84ER45076.

\section{REFERENCES}

1. S.S.P. Parkin, Pnys. Rev. Lett. Z1, 1641 (1993).

2. B.P. Gurney, V.S. Speriosu, J.P. Nozieres, H.F. Lefakis, D.R. Wilhoit, and D.U. Need, Phys. Rev. Lett. 21, 4023 (1993).

3. J.G. Tobin, G.D. Waddill, Hua Li, and S.Y. Tong, Phys. Rev. Lett. ZQ, 4150 (1993).

4. J.G. Tobin, G.D. Waddjll, and D.P. Pappas, Phys. Rev. I elt. 68, 3642 (1992).

5. G. Schütz, et. al., Phys. Fuv. Letr. 62. 2620 (1989).

6. F.Z. Hang, et al., Bulletin APS 22,904 (1994), R27.4; B. Sinkovic and C.S. Fadley, Phys. Rev. B31, 4665 (1985); B. Sinkovic, et al., Phys. Rev. Letr. 55, 1227 (1985); B. Hermsmeier, et. al., Phys. Rev. Lett. 62, 478 (1989).

7. M.T. Johnson, HI. Stamberg, and H.P. Hughes, J. Phys. C220, 4385 (i i987).

8. J.G. Tobin, M.K. Wagner, X.Q. Guo, and S.Y. Tong, Mat. Res. Soc. Symp. Proc. 208, 283 $\{1991\}$. 
Figure 1. MXCD-Photoelectron Diftraction:

Comparison of Experiment and Theory for $4 \mathrm{ML} \mathrm{Fe} / \mathrm{Cu}(001)$

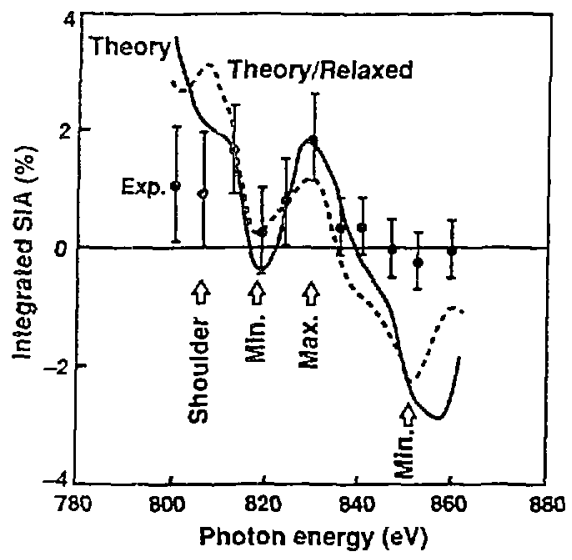

Calculated (solid curve: $d_{12}=d_{23}=1.8 \AA, T_{p}=.19$; dashed curve: $d_{12}=1.9 \AA$, $\mathrm{d}_{23}=1.7 \AA, \mathrm{r}_{\mathrm{p}}=0.17$ ) and measured intensity asymmetries along the [111] direction as a function of photon energy. The oscillatory behavior in the curves is due to spin-dependent photoelectron diffraction. Representarive error bars are included with the experimental data, shown as discrete values (triangles). See text for details. It is the positions of the minima and maxima that is the crucial variation in photoelectron diffraction. Exact quantitative agreement will require higher signal to noise and refinement of the model structure. [Note match of zero crossings.] 